\title{
Ultrasound stylet for non-image-guided ventricular catheterization
}

\author{
*Nathaniel K. Coulson, MS, ${ }^{1}$ Peter A. Chiarelli, MD, PhD, ${ }^{2}$ David K. Su, MD, ${ }^{2}$ Jason J. Chang, MD, ${ }^{2}$ \\ Brian MacConaghy, ${ }^{3}$ Revathi Murthy, MS, ${ }^{1}$ Peter Toms,${ }^{4}$ Terrence L. Robb, ${ }^{4}$ \\ Richard G. Ellenbogen, MD, ${ }^{2}$ Samuel R. Browd, MD, PhD, ${ }^{2}$ and Pierre D. Mourad, PhD ${ }^{1,3,5}$ \\ Departments of ${ }^{1}$ Bioengineering and ${ }^{2}$ Neurosurgery, ${ }^{3}$ Applied Physics Laboratory, University of Washington; ${ }^{4}$ Condor Electronics, \\ Seattle; and ${ }^{5}$ Division of Engineering and Mathematics, University of Washington, Bothell, Washington
}

OBJECT Urgent ventriculostomy placement can be a lifesaving procedure in the setting of hydrocephalus or elevated intracranial pressure. While external ventricular drain (EVD) insertion is common, there remains a high rate of suboptimal drain placement. Here, the authors seek to demonstrate the feasibility of an ultrasound-based guidance system that can be inserted into an existing EVD catheter to provide a linear ultrasound trace that guides the user toward the ventricle.

METHODS The ultrasound stylet was constructed as a thin metal tube, with dimensions equivalent to standard catheter stylets, bearing a single-element, ceramic ultrasound transducer at the tip. Ultrasound backscatter signals from the porcine ventricle were processed by custom electronics to offer real-time information about ventricular location relative to the catheter. Data collected from the prototype device were compared with reference measurements obtained using standard clinical ultrasound imaging.

RESULTS A study of porcine ventricular catheterization using the experimental device yielded a high rate of successful catheter placement after a single pass (10 of 12 trials), despite the small size of pig ventricles and the lack of prior instruction on porcine ventricular architecture. A characteristic double-peak signal was identified, which originated from ultrasound reflections off of the near and far ventricular walls. Ventricular dimensions, as obtained from the width between peaks, were in agreement with standard ultrasound reference measurements $(p<0.05)$. Furthermore, linear ultrasound backscatter data permitted in situ measurement of the stylet distance to the ventricular wall $(p<0.05)$, which assisted in catheter guidance.

CONCLUSIONS The authors have demonstrated the ability of the prototype ultrasound stylet to guide ventricular access in the porcine brain. The alternative design of the device makes it potentially easy to integrate into the standard workflow for bedside EVD placement. The availability of a fast, easy-to-use, inexpensive guidance system can play a role in reducing the complication rate for EVD placement.

http://thejns.org/doi/abs/10.3171/2015.2.PEDS14387

KEY WORDS cerebrospinal fluid; hydrocephalus; ventriculoperitoneal shunts; external ventricular drainage; ultrasound; technique

$\mathrm{P}$ LACEMENT of an external ventricular drain (EVD) is one of the most common urgent neurosurgical procedures there is. Indications include treatment of elevated intracranial pressure secondary to subarachnoid hemorrhage, intraventricular hemorrhage, meningitis, traumatic cerebral edema as well as failure of an existing CSF diversion. A ventriculostomy is routinely performed at bed- side, utilizing external anatomical landmarks of the skull to guide catheter trajectory. Suboptimal final placement of EVDs occurs in 2\%-22\% of cases at high-volume centers, ${ }^{13,16,35,43}$ and such misplaced catheters require replacement due to low output in $3.8 \%-11 \%$ of total cases..$^{14}$ Accurate freehand placement into the desired ventricle occurs in $40 \%-85 \%$ of attempts when a targeted location is com-

ABBREVIATIONS EVD = external ventricular drain; $\mathrm{I}_{\text {SPTA }}=$ spatial peak temporal average; $\mathrm{PMN}-\mathrm{PT}=$ magnesium niobate-lead titanate; $\mathrm{PRF}=$ pulse repetition frequency. SUBMITTED July 25, 2014. ACCEPTED February 4, 2015.

INCLUDE WHEN CITING Published online July 3, 2015; DOI: 10.3171/2015.2.PEDS14387.

DISCLOSURE All authors received financial support for this project from the University of Washington Center for Commercialization and the Coulter Foundation. Drs.

Mourad and Browd have a financial interest in this technology, which a company they co-founded has licensed from the University of Washington. Mr. Coulson received a

fellowship from the Mary Gates Foundation. Dr. Su received support from an NIH training grant (R25 NS079200).

* Mr. Coulson and Dr. Chiarelli contributed equally to this work. 
pared to the ultimate catheter location. ${ }^{20,21,23,43}$ Particularly in patients with irregular anatomy, multiple catheter passes can produce significant brain trauma, intracranial hemorrhage, delays in treatment, and suboptimal CSF diversion. The complication rate from ventriculostomy placement can be as high as $26 \%,{ }^{27}$ and significant risk factors for unsatisfactory placement include small ventricular size and midline shift. ${ }^{14}$

The standard technique for frontal ventricular catheter insertion has been described at length and involves insertion of the catheter at Kocher's point. ${ }^{36,46}$ Positioning the distal catheter tip segment within the lateral ventricle near the foramen of Monro relies on an accurate trajectory inferred from external landmarks and an orthogonal angle to the skull. The choice of a perpendicular trajectory can be hindered by incomplete visualization of the tangential plane, surgical drapes obscuring external landmarks, and movement of the patient under conscious sedation. Differing cranial morphology across patient populations is a widely recognized phenomenon that may further complicate the relationship between the external calvarial surface and the foramen of Monro. ${ }^{6,8,10,18,26}$ Popular assistive technologies intended to improve catheter trajectory have included the use of tripods (i.e., Ghajar Guide), fiberoptic endoscopy, and synchronized image guidance. The Ghajar Guide, while highly portable, allows only a fixed-angle path toward a position in the ventricle that is inferred from the skull surface. $28,29,31$ Endoscope technology provides confirmation of ventricular entry but does not assist with the choice of initial trajectory. ${ }^{22,33,41,42} \mathrm{CT}$ or MR image guidance is useful for the dynamic choice of trajectory, yet involves a relatively long setup time, and portability of equipment outside of the operating room can be a challenge. ${ }^{11,15,18,45}$ Furthermore, such image guidance relies on a static prior image rather than a current picture of ventricular boundaries. Finally, ultrasound image guidance requires a craniotomy for its use, a large hole relative to that needed for catheter insertion, with attendant additional time, and training in the use of diagnostic ultrasound machine, as well as the machine's presence during the procedure. Given the existing options, an ideal technology for catheter guidance would 1) be portable, for use in a range of settings, 2) allow dynamic choice of trajectory, 3) facilitate rapid setup, and 4) provide guidance based on real-time features of the immediately existing ventricular architecture.

Here we address the need for a practical and fast solution to ventricular catheter guidance consistent with existing neurosurgical workflow. We have designed and constructed an ultrasound catheter stylet capable of displaying ventricular proximity and cross-sectional ventricular width in real-time during catheter insertion. A thin ultrasound beam is emitted from the tip of the stylet and illuminates the proximal and distal ventricular interfaces, producing characteristic reflection peaks that can inform choice of catheter angle. We assess the performance of this experimental stylet by comparing measured porcine ventricular dimensions against simultaneously acquired 2D reference ultrasound images. We successfully performed pilot tests, demonstrating the ability of our ultrasound stylet to yield 1-pass ventricular catheterization in a porcine model.

\section{Methods}

\section{Stylet Design and Construction}

The ultrasound stylet has a fish-mouth opening at its distal tip and has dimensions equal to those of existing commercial catheter stylets. This allows its use with existing clinical catheter designs, without adaptation. The ultrasound stylet consists of a $20-\mathrm{cm}$-long hollow tube of surgical-grade stainless steel with an outer diameter of $1.65 \mathrm{~mm}$, which is interchangeable with standard 18-gauge catheter stylets. The ultrasound-emitting element placed at the distal tip of the tube is secured with conductive epoxy (Ablestik Eccobond 56C, cat \#9), with the insulated wires that power the transducer placed within the hollow stylet and connected first to an electrical matching network then to external controlling electronics, described below (Fig. 1).

The transducer design is constrained in the aperture to a diameter of $1.3 \mathrm{~mm}$ and a thickness of $0.2 \mathrm{~mm}$ (Fig. 1). The transducer's piezoelectric element consists of lead magnesium niobate-lead titanate (PMN-PT). The thickness of the element corresponds to the thickness mode resonance at $10 \mathrm{MHz}$, optimizing the device for sending and receiving $10 \mathrm{MHz}$ ultrasound. A quarter-wave matching layer (measuring $0.048 \mathrm{~mm}$ in thickness) of conductive Ablestik epoxy was applied to the front face of the transducer. Electrical connections were made using the same conductive epoxy as the matching layer. The epoxy matching later acted as a ground electrode and also minimized the acoustic impedance difference between the transducer material and the body. This design, and air placed behind the transducer, facilitated maximum transmission of the ultrasound from the transducer to the brain when the stylet tip was placed on or within the brain.

\section{Device Properties}

We chose $10 \mathrm{MHz}$ for the frequency of the transducer, to optimize penetration depth and directional sensitivity. As the resonant frequency of the piezoelectric element increases, the maximum penetration depth of the ultrasound decreases. Decreasing the resonant frequency increases the beam spread, thus reducing directional sensitivity. At the chosen frequency of $10 \mathrm{MHz}$, the ultrasound wave is able to penetrate brain at sufficient depth to reach the human ventricles $(5 \mathrm{~cm})$, while the beam remains sufficiently narrow to optimize angular sensitivity. Figure 2 is a field simulation of the transducer, demonstrating the effect of variable frequency on the dispersion and penetration depth of the resulting beam. The device operated at a pulse repetition frequency (PRF) of $2.5 \mathrm{kHz}$ with eight $10-\mathrm{MHz}$ cycles per pulse. The device reported only ultrasound backscatter amplitude versus time information, which was subsequently translated to amplitude versus distance information using the speed of sound in brain tissue $(1540 \mathrm{~m} / \mathrm{sec})$. This nonimaging modality is known as A-mode or A-line ultrasound.

Device output pressure and intensity were measured using a calibrated Onda HNR-1000 needle hydrophone (Onda Corporation) submerged, along with the stylet, in degassed water. A Lecroy Waverunner oscilloscope (model LT322, Teledyne LeCroy) was used to display and record the pressure measured by the needle hydrophone. 


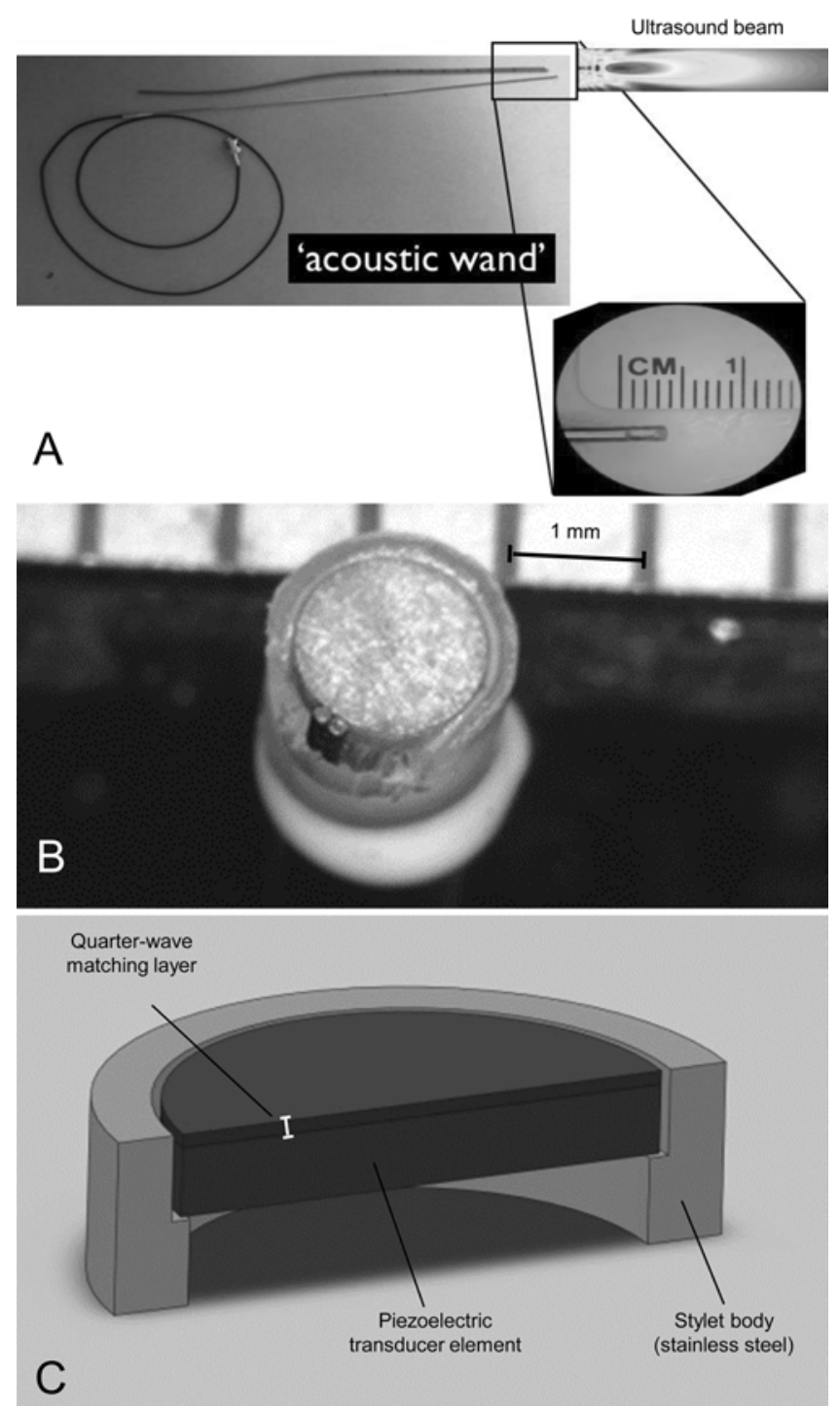

FIG. 1. Photographs and illustration of the stylet and transducer tip. A: The current iteration of the stylet shown next to a clinical catheter, with a reference image for device scale. A sample beam is displayed at the tip of the stylet to delineate the approximate field direction (not to scale). B: The mounted lead PMN-PT transducer is shown with exposed wires prior to making the electrical connections with conductive epoxy. C: The PMN-PT element is shown in a cutaway view to illustrate the design and housing of the transducer.

During in vivo experiments, our custom electronics provided power to the transducer. In vitro measurements of device output pressure used an Electronics and Innovation amplifier (model A150) controlled by an Agilent Technologies function generator (model 33220A). This allowed characterization of the device output over a larger range of ultrasound parameters than achievable with our custom electronics. Maximum pressure was measured at the spatial ultrasound peak in the far field of the transducer. Spatial peak temporal average $\left(\mathrm{I}_{\mathrm{SPTA}}\right)$ intensities of no greater than $45 \mathrm{~mW} / \mathrm{cm}^{2}$ were applied during the experiments, with typical $\mathrm{I}_{\mathrm{SPTA}}$ values near $10 \mathrm{~mW} / \mathrm{cm}^{2}$. These values fall well below the $94 \mathrm{~mW} / \mathrm{cm}^{2}$ FDA limit of ultrasound for cephalic imaging. ${ }^{9}$

\section{Electronics Design}

The device we have constructed has 4 major parts: an ultrasound pulser, transmit/receive switch, bandpass filters, and a LeCroy Waverunner LT322 oscilloscope (Fig. $3)$. The oscilloscope was used to visualize the signal generated by the device during prototype testing. The Supertex MD ultrasound pulse generator (model 1822DB3) received direct-current voltage from its power supply, creating electrical pulses with a carrier frequency of 10 $\mathrm{MHz}, \mathrm{PRF}$ of $2.5 \mathrm{kHz}$, at 8 cycles per pulse, and peak voltage values beyond the $20 \mathrm{~V}$ we used in our studies. The MD0100 TX/RX chip (Analog Devices Inc.) allowed emission by the pulser of high voltage to the transducer and collection of low-voltage signals from the transducer. An ADA4817 2nd-order band pass chip with a 3-dB bandwidth was used to reduce excess noise accumulated in the systems electronics, increasing the signal to noise ratio of the device. Throughout this study, only the LeCroy oscilloscope was used to visualize ultrasound backscatter data from the stylet.

\section{In Vivo Experimental Procedure and Device Use}

All procedures conformed to national guidelines and were approved by the University of Washington's Institutional Animal Care and Use Committee. A porcine model was used for in vivo testing of ventricular catheter insertion. All pigs were farm-type, with an average body weight of $40 \mathrm{~kg}$, approximate body length $4 \mathrm{ft}$. Animal studies were performed in a large-animal surgical suite. We used a combination of recently killed and living pigs during the experiments. In vivo experiments used isoflurane anesthesia, which was continuously monitored by a certified veterinarian. Pigs from which cadaveric specimens were to be taken were killed immediately prior to our study, after their use for peripheral procedures (e.g., abdominal laparoscopy). The catheterization procedure was the same for both cadaveric and live pigs. A $6 \times 6-\mathrm{cm}$ craniotomy was created using a perforator drill and craniotome (Stryker), with the medial extent approximately $3-4 \mathrm{~mm}$ from the midline and the posterior edge $10 \mathrm{~mm}$ anterior to the bregma. A SonoSite Micromaxx portable ultrasound imaging device (SonoSite Inc.) with a 25-mm, 6-13-MHz, linear array L25e transducer was used to monitor location of the stylet tip within the brain. The dura was coagulated using bipolar electrocautery and subsequently incised. The underlying arachnoid/pia were correspondingly cauterized and perforated using a No. 11 scalpel blade.

Equipment was situated around the subject to enable free movement of the stylet and provide the surgeon an unobstructed view of the oscilloscope display (Fig. 4). The sonographer was positioned opposite the surgeon and had a view of the SonoSite and oscilloscope displays. The prototype ultrasound-guided catheter stylet was placed on the intact dura over the expected point of entry. The standard ultrasound probe was placed on the surface of the brain in the plane of insertion of the catheter stylet. Ultrasound coupling gel was applied to the area of contact of both devices to minimize noise.

The initial study was designed to assess the ability of the stylet to measure the ventricular depth and width. The stylet was positioned $1.5 \mathrm{~cm}$ anterior to the central sulcus 

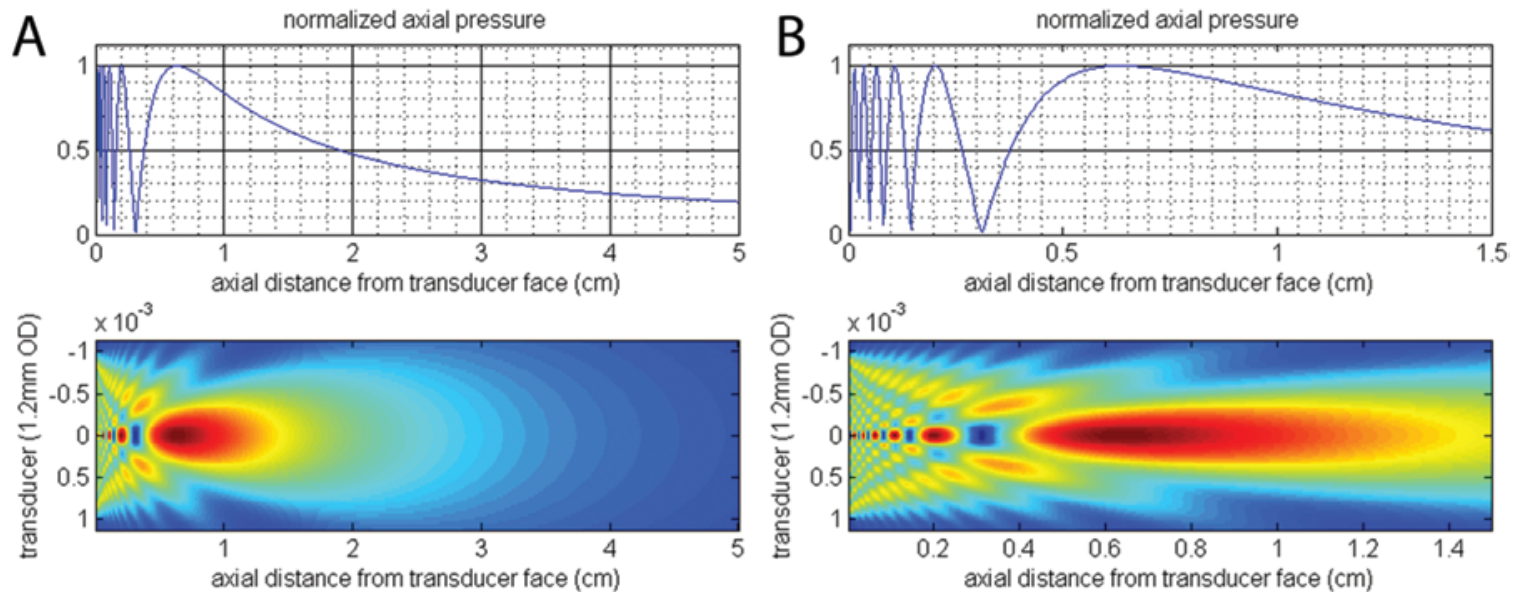

FIG. 2. Simulations showing pressure as a function of distance from the ultrasound transducer. A: Line plot showing the calculation of the spatial pattern of pressure generated by a $10-\mathrm{MHz}$ point source, out to $5 \mathrm{~cm}$ beyond the transducer. B: The same simulation as $\mathrm{A}$ but simulated to $1.5 \mathrm{~cm}$ beyond the transducer to highlight the details of the pressure field. The line plots show the pressure value as a function of distance from the transducer through the point of maximum pressure in the center of the color plots (bottom). The color plots show the spatial pattern of the pressure field, with red being the highest and blue the lowest pressures. Both point sources are calculated with the same input power and in the same medium. Figure is available in color online only.

(determined by SonoSite ultrasound) and $1 \mathrm{~cm}$ lateral to midline, and aimed perpendicular to the surface of the brain. When aiming the stylet toward the ventricle, 2 Aline ultrasound peaks were apparent, likely arising from the proximal and distal interfaces between brain and ventricle along the catheter trajectory. As there is little meaningful impedance change within ventricular CSF, the 2 noted peaks were separated by a region of low reflection intensity. Measurements of the depth to the proximal brain-ventricle interface, as well as the ventricular width along the catheter trajectory, were obtained both with the A-line ultrasound device and the SonoSite imaging device.

A second study was designed to test blinded use of the ultrasound stylet. The stylet operator was instructed to use the presence or absence of the 2-peak signal on the oscilloscope to indicate that the catheter was aimed at a ventricular target. The operator was told to initially aim the catheter orthogonal to the brain surface, and to then correct the trajectory to maximize the peak-to-peak separation of the A-line doublet, a characteristic of the trace that was predicted to approximate an angle aimed at the foramen of Monro. Lastly, the subject was informed that as the ventricle was approached, the double-peak feature

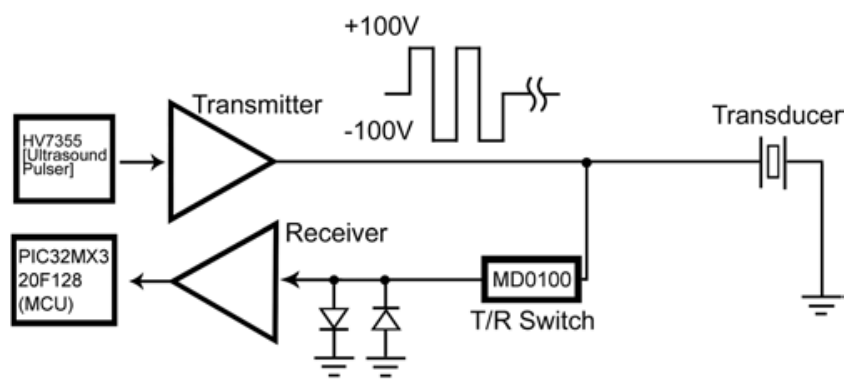

Application Circuit

FIG. 3. A block diagram of the circuit components highlighting signal path. A detailed description of each component is provided in the Electronics Design section. MCU = microcontroller unit. would shift closer to the graphical origin on the oscilloscope. This set of instructions allowed the user to guide the placement of the stylet tip into the ventricle, without inspection of the 2D ultrasound reference image at any point. Static images were collected from the SonoSite to assess the placement of the tip of the ultrasound stylet at various time points during the procedure.

\section{Data Analysis}

Measurements of ventricular depth and width were obtained from the SonoSite images using ImageJ software

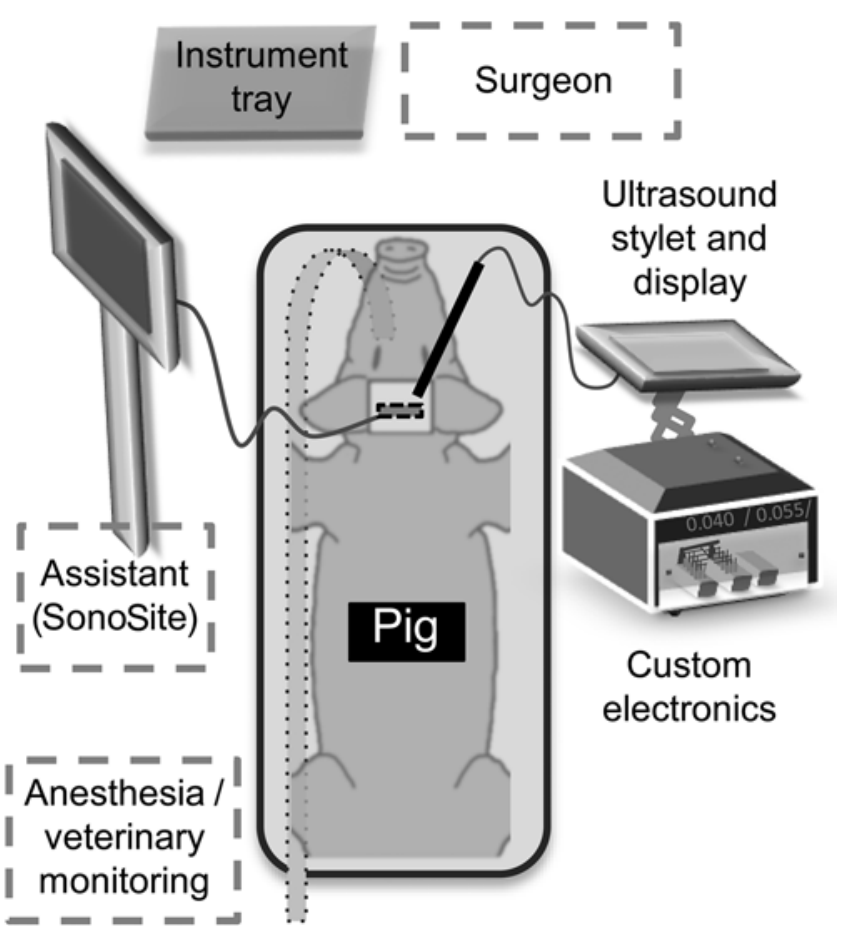

FIG. 4. A diagram of the experimental setup with equipment and personnel labeled. 
(National Institutes of Health). An image scale was set (pixels $/ \mathrm{mm}$ ) using the native scale of raw 2D ultrasound images as a reference. Distance was measured from the stylet tip at the external brain surface to the nearest wall of the ventricle along the entry trajectory. Width was recorded by measuring from the near to far ventricular walls, also along the entry trajectory. Analysis of acoustic wand measurements was performed using ImageJ and involved the determination of temporal distance (in microseconds) from the origin $(t=0)$ to the first ventricular reflection, as well as the distance between peaks. The approximate speed of sound in brain tissue was used to convert temporal measurements to distance in $\mathrm{mm}$. Distance values in their native state represented the round-trip path traversed by the ultrasound pulse, and were necessarily divided by a factor of 2. Simultaneously acquired SonoSite and prototype measurements of distance and width were compared by linear regression. A paired, 2-tailed t-test was used to analyze data from the 2 measurement techniques.

\section{Results}

\section{Measures of Ventricular Depth and Width}

Initial testing of our device involved quantification of the ventricular architecture as observed via the prototype ultrasound stylet. Figure 5A shows a representative SonoSite image, with the ventricular appearance in coronal section, along the trajectory of anticipated catheter insertion. The distance measurement from the brain surface to the point of ventricular entry and the ventricular width along the catheter trajectory are also described. Figure 5C provides an example A-line signal trace from the prototype acoustic wand, placed on the brain surface and directed at the perpendicular insertion angle. The ultrasound reflection from the skull base is seen as a large peak at 40 $\mu \mathrm{sec}(60 \mathrm{~mm})$, which provides a reassuring point of reference regarding the overall calvarial depth. Paired peaks are seen at 19 and $21 \mu \mathrm{sec}$, corresponding to depths of 28.5 and $31.5 \mathrm{~mm}$, respectively. These peaks arise from reflections at the near and far ventricular interfaces and are displayed in expanded view in Fig. 5D.

Figure 6 displays a comparison of ventricular depth, as measured with the acoustic wand and the reference ultrasound system. Data points were obtained from coincidently acquired stylet and SonoSite recordings, incorporating multiple catheter insertion depths and pooled across 3 porcine trials. Linear regression analysis provided an $\mathrm{R}^{2}$ value of 0.9956 and a slope of $0.98 \pm 0.02$, indicating that the prototype device provides an accurate distance to the target, regardless of the parenchymal depth at which the measurement was obtained. Paired Student t-test applied to the comparison of stylet and SonoSite measurements yielded $\mathrm{p}<0.05$, showing that the data collection methods did not significantly differ from one another. The interpeak separation from the linear ultrasound trace was found to accurately predict the ventricular width along the insertion trajectory, as measured by ultrasound imaging (Fig. 7). A high correlation coefficient $\left(\mathrm{R}^{2}=0.9734\right)$ and low Student t-test value $(\mathrm{p}<0.05)$ were again observed, with a slope of $1.00 \pm 0.06$ between experimental probe and SonoSite data. Our results demonstrate that the pro- totype acoustic wand can both probe the depth to the ventricular target in a dynamic fashion and provide feedback regarding ventricular width as the catheter is inserted.

\section{Guidance Trials}

To further gauge the utility of our experimental device to assist in ventricular catheterization, a series of 12 guidance trials were performed. Of these trials, 3 were performed by a practicing neurosurgeon, 2 by 4th-year neurosurgery residents, and the remainder by research team members with no prior neurosurgery experience. Beyond an understanding of conventional ventricular anatomy, no individual was provided with additional details regarding location and dimensions of the porcine ventricles. The experimental trajectory of the device was chosen based on the angle that produced the greatest peak-to-peak separation of the characteristic ultrasound doublet (corresponding to the long ventricular cross-section when aiming at the foramen of Monro), while maintaining a roughly orthogonal angle to the brain surface. A successful trial was defined as a single pass cannulating the ipsilateral frontal horn of the lateral ventricle without reviewing conventional ultrasound imaging or seeking advice from team members. All 3 trials performed by our practicing neurosurgeon resulted in successful cannulation of the lateral ventricle of interest using only the prototype device for guidance. Six of the 7 trials performed by research team members were successful in accurately placing the catheter on the first attempt. One of the 2 trials performed by a neurosurgery resident was successful, although in the unsuccessful trial, no signal matching the characteristic reflection pattern of the ventricle was located and no insertion was attempted. In total, 10 of the 12 trials performed resulted in accurate placement of the catheter in the lateral ventricle in a single pass.

During guidance trials, we found that directing the stylet laterally away from the ventricle reduced the peak intensity as well as the peak-to-peak separation. As the ventricular edge was approached and subsequently passed, the 2-peak signal disappeared. Intentionally aiming the acoustic wand medially beyond the optimal angle (i.e., the angle at which the ventricular cross-section was greatest) yielded reduced peak-to-peak separation once again. We found that directing the device toward the contralateral ventricle did not yield an additional peak from CSF in the interhemispheric fissure, likely due to the close proximity of the medial porcine hemispheric surfaces. In the human, we would expect the larger interhemispheric separation to generate an additional peak, complicating the ultrasound profile. Despite the lack of a specific indicator for contralateral trajectory, such contralateral placement was avoided using the instructions for catheter placement described above (i.e., maximizing the peak-to-peak separation of the signature doublet). As the distance between the catheter and the ventricular target decreased, the distance between the 2-peak structure and the tip of the stylet reduced, as expected.

\section{Discussion}

Clinical Application of the Device

The primary goal of this study was to provide initial 

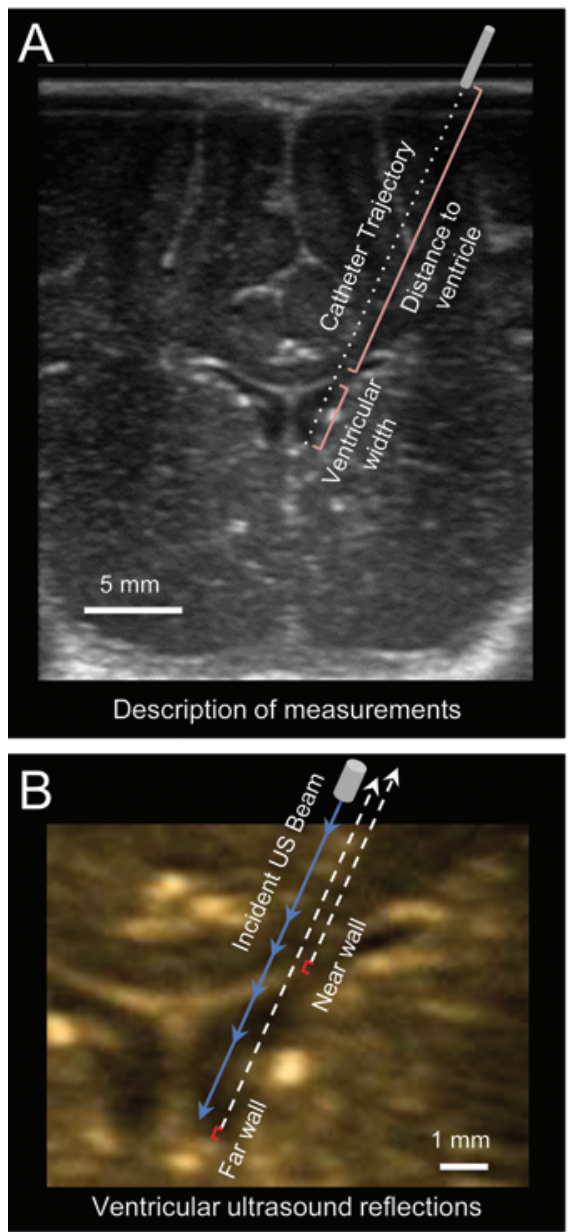

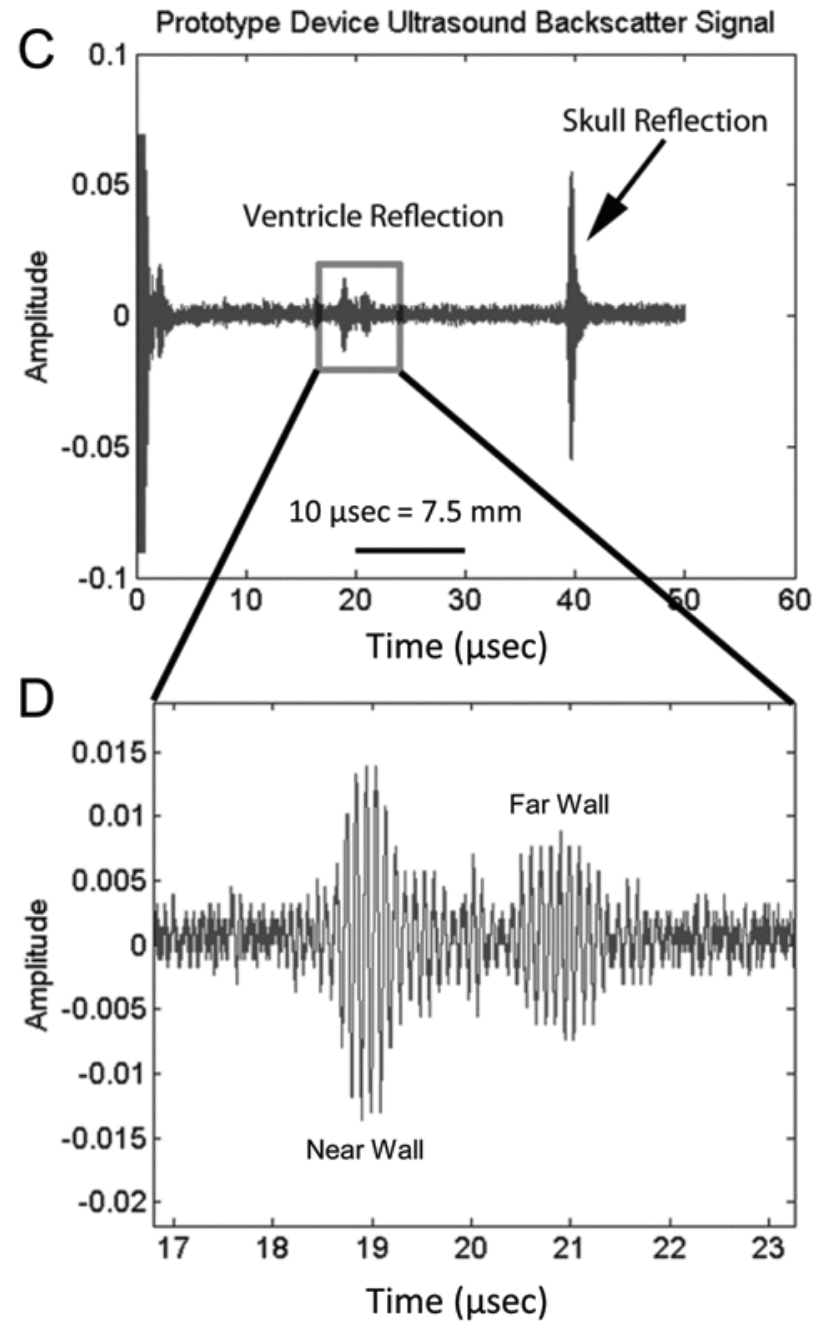

FIG. 5. A representative SonoSite image with an illustrative A-line signal trace from the prototype acoustic wand. A: A SonoSite image of a pig brain during alignment of the catheter on the brain surface shows the ultrasound trajectory and ventricular target. The measured distance to the ventricle and the ventricular width are labeled. As the stylet is advanced, the distance to the ventricle decreases, corresponding to a decrease in distance to the ventricular reflection. B: An enlarged view of the ventricular target shows ventricular walls along the entrance path that produce the signal displayed in C. C: A linear ultrasound trace acquired from the prototype device, with labeled signal components. D: Expansion of the ventricular reflection delineating the near and far ventricular wall reflections originating from the structures labeled in B. US = ultrasound. Figure is available in color online only.

assessment of a novel ultrasound stylet for ventricular catheter placement. A direct comparison of measurements taken with the prototype acoustic wand and the reference ultrasound system indicates that our stylet can successfully characterize the porcine ventricular depth and width in real-time during catheter insertion. Additionally, we have demonstrated that research team members were able to identify a ventricular target and guide the catheter into the ipsilateral ventricle using the prototype device.

Based on the small ventricular size and lack of prior knowledge of detailed porcine neuroanatomy for our users, it is highly unlikely that blind catheterization in this setting would have been successful. No participant in our study had practiced catheter insertion or had undergone any instruction on porcine ventricular anatomy prior to participation in the catheterization attempt. Particular to our study, the ventricular cavities tend to be significantly smaller in pigs than in humans; we observed an anterior horn width in pigs of 1-2 mm. The anterior horn width of preterm human neonates has been measured at 0-2.9 mm. ${ }^{5}$ Therefore, the porcine model might best represent a "slit ventricle" case in an adult human patient. Our results provide a positive initial trial and highlight the value of further refinement for this system. Future trials would benefit from the simulation of more complex pathology (e.g., midline shift or intraventricular hemorrhage) to test the capacity for our device to reliably guide ventricular access.

The ultrasound-assisted ventricular access stylet that we have described here is intended to improve accuracy of catheter placement. For the application of emergent ventricular drains, time is a critical factor, and we have considered ease-of-setup as a key design constraint in our system. We envision a clinical version of our device as a handheld stylet with accompanying electronics, including small screen, contained in a lightweight assembly located at the proximal end of the catheter. An alternative design 


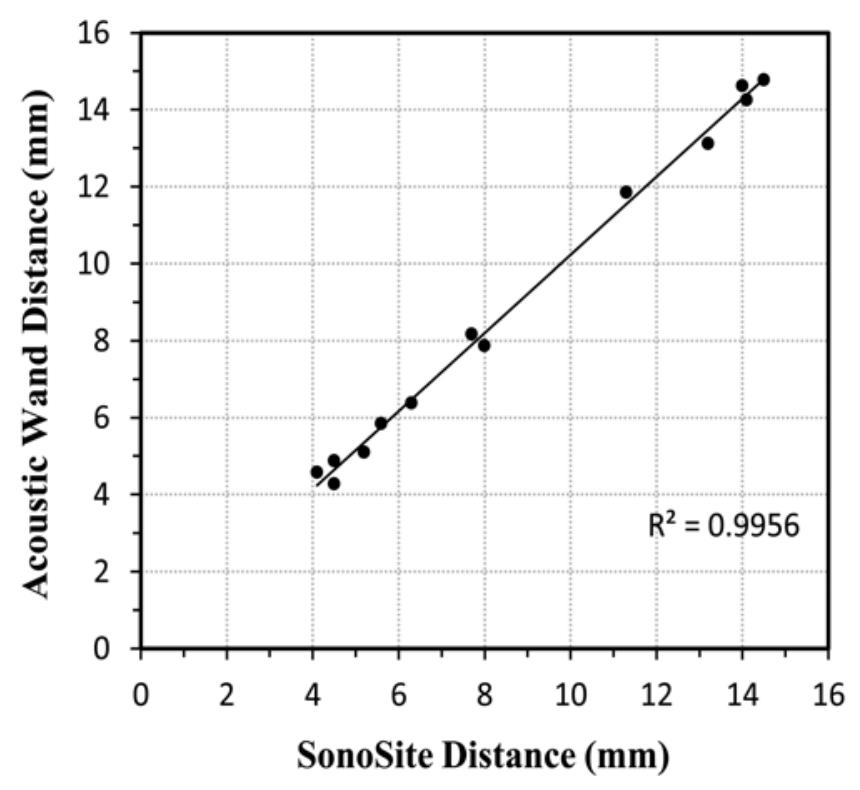

FIG. 6. A comparison between the distance measured by the SonoSite Micromaxx (x-axis) and that measured by the guided prototype (y-axis) to the nearest edge of the lateral ventricular target. Each data point represents a single measurement from each device.

would put the electronics and small screen within a cellphone sized device connected via a light-weight cable, placed adjacent to the patient and within the neurosurgeon's field of view. The stylet itself will deploy within a standard ventricular catheter with a slit or fish mouth at its distal end, and would require no additional calibration. The full system (stylet within catheter, accompanying electronics) would be small enough to be housed in a hospital ventricular access cart, and would be ready to use after removal from sterile packaging. The interpretation of a 2-peak ventricular signature on the screen would indicate to the user that the path leads toward a ventricular target. We expect that the setup of this device would add little additional time, beyond pressing an 'on' button. A decrease in the number of incorrect passes may compensate for any potential time loss during setup.

\section{Comparison With Existing Technology and Device Limitations}

Among existing assist devices for ventricular catheter placement, the Ghajar Guide is a portable and inexpensive instrument approximating a perpendicular trajectory from the skull or scalp. Once the tripod is positioned over the site of entry, a central tube guides the catheter orthogonally to the local spherical curvature. In a randomized prospective trial by O'Leary et al., the Ghajar Guide resulted in fewer passes (1.1 vs 1.5), more accurate placement at the level of the foramen of Monro, and fewer catheters crossing midline (4\% vs 33\%). ${ }^{28}$ Limitations of the guide include its inability to compensate for midline shift or abnormal ventricular anatomy, and inherent obstacles to situating the device over the access point. Although the Ghajar Guide is accurate and dependable, 2 separate surveys demonstrate that very few neurological surgeons use this guide routinely. ${ }^{29,31}$ In patients with slit ventricles, the majority of

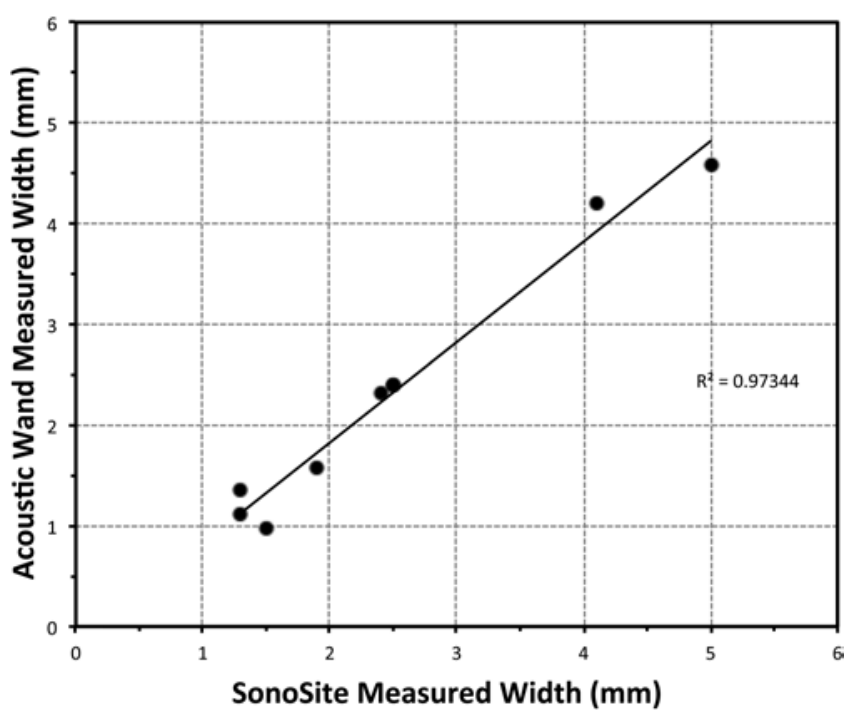

FIG. 7. A comparison of the distance between the ventricle walls (ventricle width) as measured by the SonoSite Micromaxx (x-axis) and the guided prototype (y-axis). Each data point represents a single measurement from each device.

neurosurgeons (51.9\%) use upfront image guidance, in part because of the anticipated difficulty.

In many situations of challenging catheter placement, frameless stereotactic image-guidance systems are used. An improvement in placement accuracy, resulting in more reliable proximal shunt patency rates, has been demonstrated with image guidance. ${ }^{11,15,19,45}$ These systems provide realtime visualization of catheter trajectory, although cumbersome detector components make the use of these systems impractical outside the operating room. Electromagnetic or infrared interference with the detector can occur, complicating the use of such an apparatus. Notably, these technologies synchronize the stylet to a historical static image of the supine patient's brain. Dynamic factors including accumulation of CSF, CSF leak, worsening edema, positional differences, removal of cranial bone, and replacement of cranial bone can substantially alter anatomical location of the ventricular wall and the midline septum. Such changes may unavoidably occur during the time between baseline image acquisition and catheter placement, contributing to an additional degree of variability.

Beyond infrared and inductive-coupled magnetic tracking systems, groups have used fiberoptic endoscope technology and robotic assistance for catheter placement. $22,24,33,40,41$ Thomale et al. have described a fixedframe guidance system comprised of a rigid arc, catheter guide tube, and coordinates calculated with smartphone software analyzing an uploaded DICOM image..$^{42}$

To date, ultrasound-image assisted ventricular catheter placement has predominantly occurred in the intraoperative setting. 1,3,7,17,32,34,37-39 Early placement attempts using ultrasound imaging were limited to transfontanelle techniques or cases requiring removal of cranial bone to accommodate transducer placement over the brain parenchyma. ${ }^{12,30,34,37}$ Contemporary high-resolution ultrasound imaging technology has been refined, allowing smaller transducers to fit within the bur hole alongside the cath- 
eter. ${ }^{2,25,39}$ Multiple studies confirm that intraoperative ultrasound guidance can reduce the need for revisions in ventriculoperitoneal shunt placement in the acute setting when compared to studies without image guidance $(1.8 \%$ vs $6.7 \%)^{4,44,45}$

The device presented in this work is a prototype in the first phase of development. We envision the stylet as a fully hand-held system, with elimination of electronic cables making the device a more user-friendly system, facilitating its seamless integration into the common practice of experienced neurosurgeons. The device currently uses only ultrasound backscatter from ventricular boundaries to detect the ventricle. Another technique under consideration for ventricle-edge detection would focus on detection of differences in pulsatile tissue movement between brain parenchyma and ventricular fluid. A combination of multiple modalities may help increase the already considerable ultrasound contrast between brain and ventricle.

\section{Conclusions}

We have described a novel acoustic ventricular catheter stylet with an on-board ultrasound source, whose narrow, nonimaging beam can detect the boundaries of the lateral ventricle. Analysis of the ultrasound backscatter allowed detection of the depth and width of the lateral ventricles from the distance of a twist-drill access point. With the ventricle in view, 2 high-amplitude reflections corresponding to the near and far walls of the ventricle appear. Advancing the catheter caused the paired reflections to move progressively closer to the zero point of the display, providing a means of gauging distance from ventricular entry.

This device offers an advantage over existing technologies by relying on real-time information derived from current anatomy, rather than utilizing a static picture from a previous time point. The successful function of this stylet represents a significant step in the development of a fully enclosed, miniaturized electronic system for ultrasoundassisted ventriculostomy placement. Precise catheter placement has high development potential with major impact on safe surgical care and cost savings.

\section{Acknowledgments}

We gratefully acknowledge the help of Valerie Carricaburu, $\mathrm{PhD}$, Kathie Jordon, PhD, and Rachael Hagen, MS, for substantial guidance during the entire extent of this work.

\section{References}

1. Babcock DS, Barr LL, Crone KR: Intraoperative uses of ultrasound in the pediatric neurosurgical patient. Pediatr Neurosurg 18:84-91, 1992

2. Berger MS: Ultrasound-guided stereotaxic biopsy using a new apparatus. J Neurosurg 65:550-554, 1986

3. Chandler WF, Knake JE, McGillicuddy JE, Lillehei KO, Silver TM: Intraoperative use of real-time ultrasonography in neurosurgery. J Neurosurg 57:157-163, 1982

4. Crowley RW, Dumont AS, Asthagiri AR, Torner JC, Medel $\mathrm{R}$, Jane JA, Jr, et al: Intra-operative ultrasound guidance for the placement of permanent ventricular cerebrospinal fluid shunt catheters: a single center historical cohort study. World Neurosurg 81:397-403

5. Davies MW, Swaminathan M, Chuang SL, Betheras FR: Reference ranges for the linear dimension of the intracranial ventricles in preterm neonates. Arch Dis Child Fetal Neonatal Ed 82:F218-F223, 2000

6. Dean D, Bookstein FL, Koneru S, Lee JH, Kamath J, Cutting CB, et al: Average African American three-dimensional computed tomography skull images: the potential clinical importance of ethnicity and sex. J Craniofac Surg 9:348-359, 1998

7. Enzmann DR, Irwin KM, Fine M, Silverberg GM, Hanbery JW: Intraoperative and outpatient echoencephalography through a burr hole. Neuroradiology 26:57-59, 1984

8. Eskandary H, Nematollahi-mahani SN, Zangiabadi N: Skull indices in a population collected from computed tomographic scans of patients with head trauma. J Craniofac Surg 20:545-550, 2009

9. Food and Drug Administration: Information for Manufacturers Seeking Marketing Clearance of Diagnostic Ultrasound Systems and Transducers. Rockville, MD: Food and Drug Administration, 2008

10. Haas LL: Roentgenological skull measurements and their diagnostic applications. Am J Roentgenol Radium Ther Nucl Med 67:197-209, 1952

11. Hayhurst C, Beems T, Jenkinson MD, Byrne P, Clark S, Kandasamy J, et al: Effect of electromagnetic-navigated shunt placement on failure rates: a prospective multicenter study. J Neurosurg 113:1273-1278, 2010

12. Heussinger N, Eyüpoglu IY, Ganslandt O, Finzel S, Trollmann R, Jüngert J: Ultrasound-guided neuronavigation improves safety of ventricular catheter insertion in preterm infants. Brain Dev 35:905-911, 2013

13. Huyette DR, Turnbow BJ, Kaufman C, Vaslow DF, Whiting BB, Oh MY: Accuracy of the freehand pass technique for ventriculostomy catheter placement: retrospective assessment using computed tomography scans. J Neurosurg 108:88-91, 2008

14. Kakarla UK, Kim LJ, Chang SW, Theodore N, Spetzler RF: Safety and accuracy of bedside external ventricular drain placement. Neurosurgery 63 (1 Suppl 1):ONS162-ONS167, 2008

15. Kestle JRW, Drake JM, Cochrane DD, Milner R, Walker ML, Abbott R III, et al: Lack of benefit of endoscopic ventriculoperitoneal shunt insertion: a multicenter randomized trial. J Neurosurg 98:284-290, 2003

16. Khanna RK, Rosenblum ML, Rock JP, Malik GM: Prolonged external ventricular drainage with percutaneous long-tunnel ventriculostomies. J Neurosurg 83:791-794, 1995

17. Knake JE, Chandler WF, McGillicuddy JE, Silver TM, Gabrielsen TO: Intraoperative sonography for brain tumor localization and ventricular shunt placement. AJR Am J Roentgenol 139:733-738, 1982

18. Koizumi T, Komuro Y, Hashizume K, Yanai A: Cephalic index of Japanese children with normal brain development. J Craniofac Surg 21:1434-1437, 2010

19. Levitt MR, O'Neill BR, Ishak GE, Khanna PC, Temkin NR, Ellenbogen RG, et al: Image-guided cerebrospinal fluid shunting in children: catheter accuracy and shunt survival. J Neurosurg Pediatr 10:112-117, 2012

20. Lind CRP, Tsai AMC, Law AJJ, Lau H, Muthiah K: Ventricular catheter trajectories from traditional shunt approaches: a morphometric study in adults with hydrocephalus. J Neurosurg 108:930-933, 2008

21. Lind CRP, Tsai AMC, Lind CJ, Law AJJ: Ventricular catheter placement accuracy in non-stereotactic shunt surgery for hydrocephalus. J Clin Neurosci 16:918-920, 2009

22. Lollis SS, Roberts DW: Robotic catheter ventriculostomy: feasibility, efficacy, and implications. J Neurosurg 108:269274,2008

23. Low D, Drake JM, Seow WT, Ng WH: Management of ventriculo-peritoneal shunts in the paediatric population. Asian J Neurosurg 5:7-14, 2010 
24. Manwaring KH: Endoscopic-guided placement of the ventriculoperitoneal shunt, in Manwaring KH, Crone KR (ed): Neuroendoscopy. New York: Mary Ann Liebert, 1992, pp 29-40

25. Masuzawa H, Kanazawa I, Kamitani H, Sato J: Intraoperative ultrasonography through a burr-hole. Acta Neurochir (Wien) 77:41-45, 1985

26. McShane D: Neurocranial form: differentiating four ethnic populations using a simple CT scan measure. Int J Neurosci 21:137-143, 1983

27. Ngo QN, Ranger A, Singh RN, Kornecki A, Seabrook JA, Fraser DD: External ventricular drains in pediatric patients. Pediatr Crit Care Med 10:346-351, 2009a

28. O'Leary ST, Kole MK, Hoover DA, Hysell SE, Thomas A, Shaffrey CI: Efficacy of the Ghajar Guide revisited: a prospective study. J Neurosurg 92:801-803, 2000

29. O'Neill BR, Velez DA, Braxton EE, Whiting D, Oh MY: A survey of ventriculostomy and intracranial pressure monitor placement practices. Surg Neurol 70:268-273, 2008

30. Phillips SB, Gates M, Krishnamurthy S: Strategic placement of bedside ventriculostomies using ultrasound image guidance: report of three cases. Neurocrit Care 17:255-259, 2012

31. Rehman T, Rehman AU, Rehman A, Bashir HH, Ali R, Bhimani SA, et al: A US-based survey on ventriculostomy practices. Clin Neurol Neurosurg 114:651-654, 2012

32. Rogers JV III, Shuman WP, Hirsch JH, Lange SC, Howe JF, Burchiel K: Intraoperative neurosonography: application and technique. AJNR Am J Neuroradiol 5:755-760, 1984

33. Roth J, Constantini S: Selective use of intra-catheter endoscopic-assisted ventricular catheter placement: indications and outcome. Childs Nerv Syst 28:1163-1169, 2012

34. Rubin JM, Dohrmann GJ: Use of ultrasonically guided probes and catheters in neurosurgery. Surg Neurol 18:143148,1982

35. Saladino A, White JB, Wijdicks EFM, Lanzino G: Malplacement of ventricular catheters by neurosurgeons: a single institution experience. Neurocrit Care 10:248-252, 2009

36. Schültke E: Theodor Kocher's craniometer. Neurosurgery 64:1001-1005, 2009

37. Shkolnik A, McLone DG: Intraoperative real-time ultrasonic guidance of ventricular shunt placement in infants. Radiology 141:515-517, 1981

38. Strowitzki M, Komenda Y, Eymann R, Steudel WI: Accuracy of ultrasound-guided puncture of the ventricular system. Childs Nerv Syst 24:65-69, 2008

39. Strowitzki M, Moringlane JR, Steudel W: Ultrasound-based navigation during intracranial burr hole procedures: experience in a series of 100 cases. Surg Neurol 54:134-144, 2000

40. Taha JM, Crone KR: Endoscopically guided shunt placement. Tech Neurosurg 1:159-167, 1996

41. Theodosopoulos PV, Abosch A, McDermott MW: Intraoperative fiber-optic endoscopy for ventricular catheter insertion. Can J Neurol Sci 28:56-60, 2001

42. Thomale UW, Knitter T, Schaumann A, Ahmadi SA, Ziegler P, Schulz M, et al: Smartphone-assisted guide for the placement of ventricular catheters. Childs Nerv Syst 29:131-139, 2013

43. Toma AK, Camp S, Watkins LD, Grieve J, Kitchen ND: External ventricular drain insertion accuracy: is there a need for change in practice? Neurosurgery 65:1197-1201, 2009

44. Whitehead WE, Jea A, Vachhrajani S, Kulkarni AV, Drake JM: Accurate placement of cerebrospinal fluid shunt ventricular catheters with real-time ultrasound guidance in older children without patent fontanelles. J Neurosurg 107 (5 Suppl):406-410, 2007

45. Wilson TJ, Stetler WR Jr, Al-Holou WN, Sullivan SE: Comparison of the accuracy of ventricular catheter placement using freehand placement, ultrasonic guidance, and stereotactic neuronavigation. J Neurosurg 119:66-70, 2013

46. Yamada SM, Yamada S, Goto Y, Nakaguchi H, Murakami $\mathrm{M}$, Hoya K, et al: A simple and consistent technique for ventricular catheter insertion using a tripod. Clin Neurol Neurosurg 114:622-626, 2012

\section{Author Contributions}

Conception and design: Mourad, Coulson, Chiarelli, Su, Chang, MacConaghy, Murthy, Ellenbogen, Browd. Acquisition of data: Mourad, Coulson, Chiarelli, Su, Chang, MacConaghy, Murthy, Toms, Robb, Browd. Analysis and interpretation of data: Mourad, Coulson, Chiarelli, Su, Chang, Robb, Browd. Drafting the article: Coulson, Chiarelli, Su, Toms, Browd. Critically revising the article: Mourad, Coulson, Chiarelli, Browd. Reviewed submitted version of manuscript: Mourad, Coulson, Chiarelli, Su, Chang, Murthy, Toms, Robb, Ellenbogen, Browd. Statistical analysis: Coulson. Administrative/technical/material support: Mourad, Murthy, Toms, Robb, Ellenbogen, Browd. Study supervision: Mourad, Ellenbogen, Browd.

\section{Correspondence}

Pierre D. Mourad, Department of Neurological Surgery, University of Washington, 1959 N.E. Pacific St., Seattle, WA 98195-6470. email: doumitt@uw.edu. 